\title{
Agreement rates for sleep/wake judgments obtained via accelerometer and sleep diary: A comparison
}

\author{
TOMOYUKI KAWADA \\ Nippon Medical School, Tokyo, Japan
}

\begin{abstract}
Agreement rates for waking and sleeping obtained via sleep diary and accelerometer were evaluated, to compare the two methods. Sleep/wake data for consecutive days and nights were surveyed in 119 healthy university students. Accelerometer sleep/wake judgments obeyed the standard algorithm. Agreement rates for waking and sleeping according to accelerometer versus sleep diary, respectively, were calculated. Sleep diary data were set as a baseline. Seventy-six subjects $(63.9 \%), 22$ to 32 years of age, presented perfect data for the analysis. The mean sleep times, in minutes, judged by sleep diary and by accelerometer, were 482.3 and 629.6 , respectively. The mean percentages and standard deviations of agreement on wake and sleep were $77.5 \%(S D=10.2)$ and $86.1 \%(S D=6.2)$, respectively. There was a significant negative relationship between the agreement rates for wake and sleep $(r=-.482, p<.01)$. The accelerometer showed some measurement failure during waking, presumably because of the decrease in body movement. Sleep diary data during daytime appear to be more valid for detecting a sleep/wake cycle than are accelerometer data. In contrast, nocturnal sleep diary data might be supplemented by the use of an accelerometer as long as participants do not have insomnia.
\end{abstract}

The evaluation of patterns of sleeping and waking is essential for the identification and management of sleep problems (Kryger, Roth, \& Dement, 2005). Procedures for the assessment of these patterns are also important in sleep research.

A frequent approach to assessing patterns of sleeping and waking is simply to ask participants about these patterns either via a questionnaire or via interview. Data can also be collected from sleep diaries, in which participants provide information regarding their sleep patterns over time. Other methods include actigraphy, in which accelerometers provide a quantitative and objective estimation of sleep times and interruptions over several days (Sadeh, Sharkey, \& Carskadon, 1994).

These methods are different in terms of duration, effort, cost, and source of information. Sleep diaries are based on subjective self-report, whereas accelerometers record sleep/wake patterns objectively by measuring body movements. Previous studies have evaluated the respective advantages and disadvantages of these methods (Kawada et al., 2001; Kawada et al., 2008; Lockley, Skene, \& Arendt, 1999). Subjective reports of sleep, as opposed to objective measurement of sleep, can be imprecise. However, subjective reports have the advantage of being more cost effective for field research (Kawada, 2002; Kawada \& Suzuki, 2002).

Previous investigation has focused more on sleep onset, sleep end, or sleep duration, divided into weekday and weekend time frames (Werner, Molinari, Guyer, \& Jenni, 2008), than on measurement of sleep via accelerometer versus diary, or measurement of waking via accelerometer versus diary. Since the accelerometer detects body movement instead of brain activity, sleep polygraphy is to be preferred in sleep study. But monitoring a sleep/wake cycle via polygraphy is methodologically difficult, especially during waking periods. Entries are made in a sleep diary after one wakes up, so there is no accurate measurement of sleep. But we can still consider the relationship between a subjective diary evaluation of sleeping and waking and an objective measurement via accelerometer.

The author intended to analyze the relationship between the two methods, using the sleep diary as a baseline. In addition, sleep/wake times in healthy young adults, according to measurements from both the sleep diary and the accelerometer, were presented.

\section{METHOD}

\begin{abstract}
Subjects
A total of 119 healthy university students (age range, 22-32 years) participated in the study. All subjects were given an orientation, and were told the purpose of the study. Forty-three subjects were dropped from the study for forgetting to make entries in the sleep diary or for forgetting to wear the accelerometer for 5 consecutive days and nights. Seventy-six of the 119 students $(63.9 \%)$ were therefore included in the final data analysis.
\end{abstract}

T.Kawada, kawada@nms.ac.jp 


\section{Accelerometer}

The subjects were directed to wear continuously an activity- and sleep-monitoring device based on a piezoelectric accelerometer, the Actiwatch (Mini Mitter Co., Sunriver, OR), on the wrists of the nondominant arms and to remove it only if they might get wet.

The sampling frequency of the Actiwatch was set at $32 \mathrm{~Hz}$ and the sensitivity at $0.05 \mathrm{~g}$, using $1 \mathrm{~min}$ as the unit; the number of body movements was calculated by summing the active electricity counts. If the count was 40 or more, we judged it as wake time. The count of 40 was adopted as the appropriate cutoff point between sleeping and waking (Benson et al., 2004). If the number of two epochs before the target epoch $\times 0.04+$ number of one epoch before the target epoch $\times 0.2+$ number of the target epoch + number of one epoch after the target epoch $\times 0.2+$ number of two epochs after the target epoch $\times 0.04$ exceeded 40, the condition was judged as wake. Using this algorithm, the agreement between the Actiwatch and the sleep polygraph was $88.4 \%$, averaged over 24 nights, and Pearson's correlation coefficient of total sleep time between Actiwatch and the sleep polygraph was .85 (Kawada, Kuroiwa, Sasazawa, Suzuki, \& Tamura, 2002).

\section{Sleep Diary}

Subjects were requested to keep a detailed sleep diary at 30-min intervals while they were being monitored by the Actiwatch. Some of the subjects made entries in their sleep diaries at 15-min intervals; we also used those data. Each subject began sleep monitoring on Monday afternoon and ended it on Friday, so we used data from Tuesday through Thursday. There was no regulation regarding the time of going to bed or waking up.

The study was approved by the local research ethics committee. As mentioned previously, subjects were told about this study, and they gave their informed written consent. Accelerometer and sleep diary data were then collected from the subjects.

\section{Statistical Analyses}

The agreement rate of sleep measured by accelerometer versus sleep reported in sleep diaries was calculated. In the same manner, the agreement rate of wake measured by accelerometer versus wake reported in sleep diaries was also calculated. All analyses were performed with two-tailed tests, and a value of $p<.05$ was considered significant. SPSS (14.0J for Windows; SPSS Japan Inc., Tokyo) was used for all statistical analyses.

\section{RESULTS}

The mean age and its standard deviation $(S D)$ were 24.7 and 1.9 years, respectively. There were 54 males $(71.1 \%)$ and 22 females $(28.9 \%)$. The mean sleep times according to sleep diaries and accelerometer were $482.3 \mathrm{~min}(S D=$ $80.4)$ and $629.6 \min (S D=125.1)$, which was a significant difference according to a matched-pair signed ranks test $(p<.001)$.

To compare the accelerometer and diary data, the agreement rate was calculated (Table 1). The average agreement rate of wake according to accelerometer versus sleep diary was $77.5 \% \pm 10.2 \%$, and the average agreement rate of sleep according to accelerometer versus sleep diary was $86.1 \% \pm 6.2 \%$. The subjects were divided into four groups by sex and age. The average agreement rates for wake and sleep among the four groups did not significantly differ.

A significant relationship between the agreement rate for wake and for sleep was recognized $(r=-0.482, p<$ .01 ) (see Figure 1), and subjects presenting good agreement rates for wake and sleep showed dramatic changes of activity during wake and sleep.
Table 1

Mean Agreement Rates for Waking and Sleeping As Assessed via Accelerometer and Sleep Diaries

\begin{tabular}{|c|c|c|c|c|c|}
\hline \multirow[b]{2}{*}{ Subjects } & \multirow[b]{2}{*}{ Number } & \multicolumn{2}{|c|}{ Wake } & \multicolumn{2}{|c|}{ Sleep } \\
\hline & & $M$ & $S D$ & $M$ & $S D$ \\
\hline Male $<25$ years of age & 24 & 78.4 & 9.4 & 86.2 & 6.6 \\
\hline$\geq 25$ years of age & 30 & 77.0 & 10.3 & 85.1 & 6.2 \\
\hline Female $<25$ years of age & 15 & 77.8 & 11.7 & 87.0 & 6.0 \\
\hline$\geq 25$ years of age & 7 & 75.7 & 10.7 & 87.5 & 5.5 \\
\hline Total & 76 & 77.5 & 10.2 & 86.1 & 6.2 \\
\hline
\end{tabular}

\section{DISCUSSION}

This cross-sectional analysis of sleep/wake agreement rates for accelerometers versus sleep diaries was calculated in healthy university students. This study provides normative data for agreement rates in a nonclinical sample.

Although we could not obtain an acceptable agreement between the two methods, the results of this study indicate that the accelerometer and the diaries measured different things (i.e., motor activity of daily life, and subjective recollections of sleep or wake times) (Carney, Lajos, \& Waters, 2004; Lockley et al., 1999), and that the data from the accelerometer and the diaries are only approximations of accurate sleep and wake times. The accelerometer and diary methods should be compared with sleep polygraphy, which - especially during sleep - should be the preferred method in such studies. Although there are no validation data for the sleep diaries using sleep polygraphy, we have previously compared the use of sleep polygraphy and the accerelometer simultaneously during a 3-h nap taken by 51 healthy male students 20 to 27 years of age. These data are unpublished. Sleep efficiency rates ranged from $30.8 \%$ to $99.4 \%$; the mean value was $77.4 \%$ ( $S D=$ $19.0 \%)$. When sleep polygraphic data were used as a standard, the mean correct agreement rate of sleep was $96.5 \%$ $(S D=3.6 \%)$. In contrast, with the accelerometer, which lacks the ability to recognize episodes of wakefulness during sleep, the mean was $23.7 \%$.

Although some of the drawbacks of this study are the absence of data based on sleep polygraphy, and the fact that the subjects were young and healthy university students who were tested only on weekdays, the sleep/wake diary is an inexpensive means of obtaining subjective information. The diary is an important baseline tool for the health-care professional when evaluating sleep problems. Information about arousal during the nocturnal period may partly be corrected by an accelerometer, given that the subjects cannot report such incidents in detail (Sadeh, 1995), although the correct agreement rate for waking was low in the present study. A negative relationship between the agreement rates for waking and sleeping, in addition to a significant difference in mean sleep time between the two methods in our study, also shows that usage of an accelerometer alone would lead to inadequate sleep/wake judgments (Paquet, Kawinska, \& Carrier, 2007).

\section{AUTHOR NOTE}

The author thanks the subjects in this study and Masao Katsumata, Hiroko Suzuki, and Takako Shimizu of the Department of Hygiene and 
A

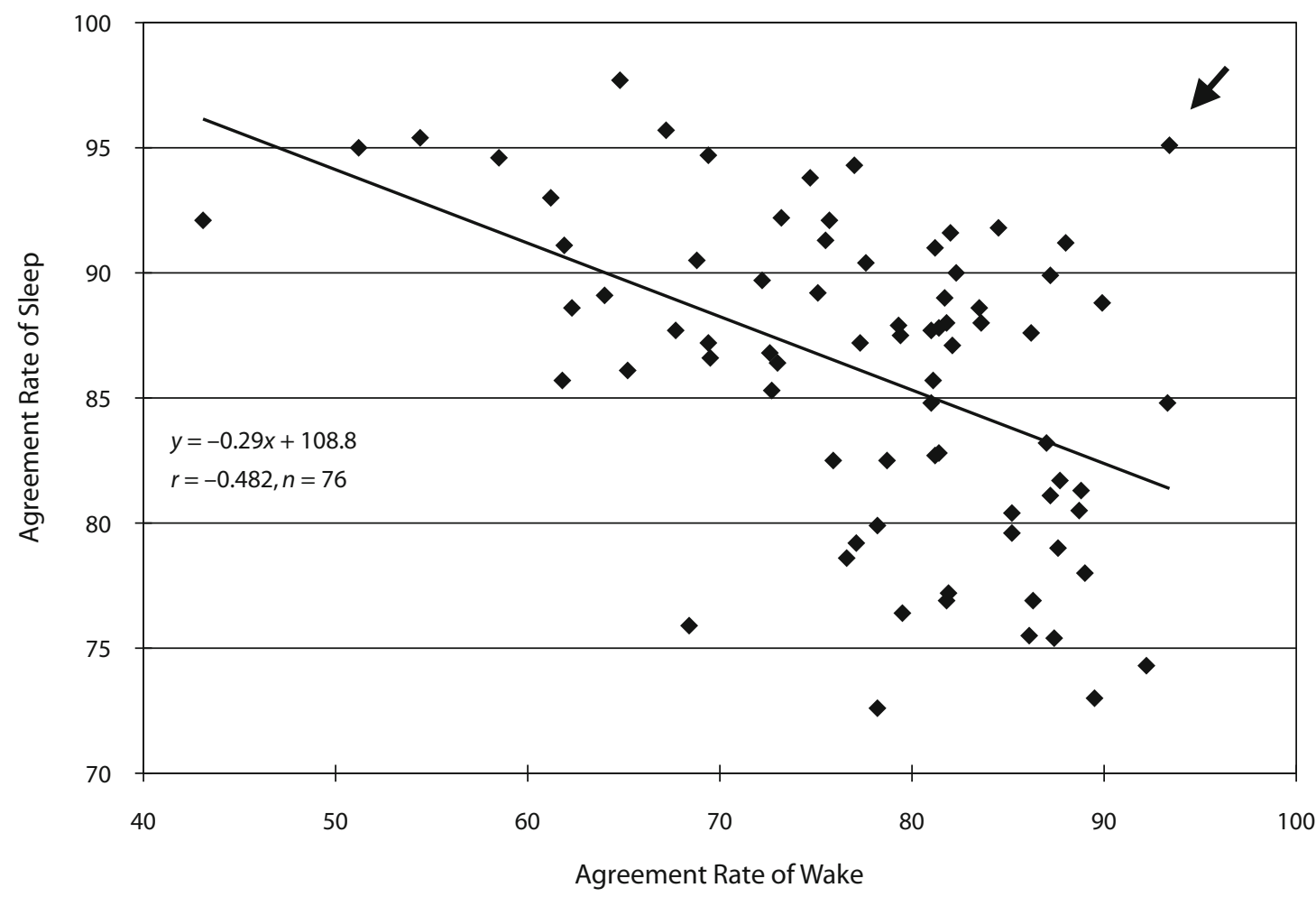

B

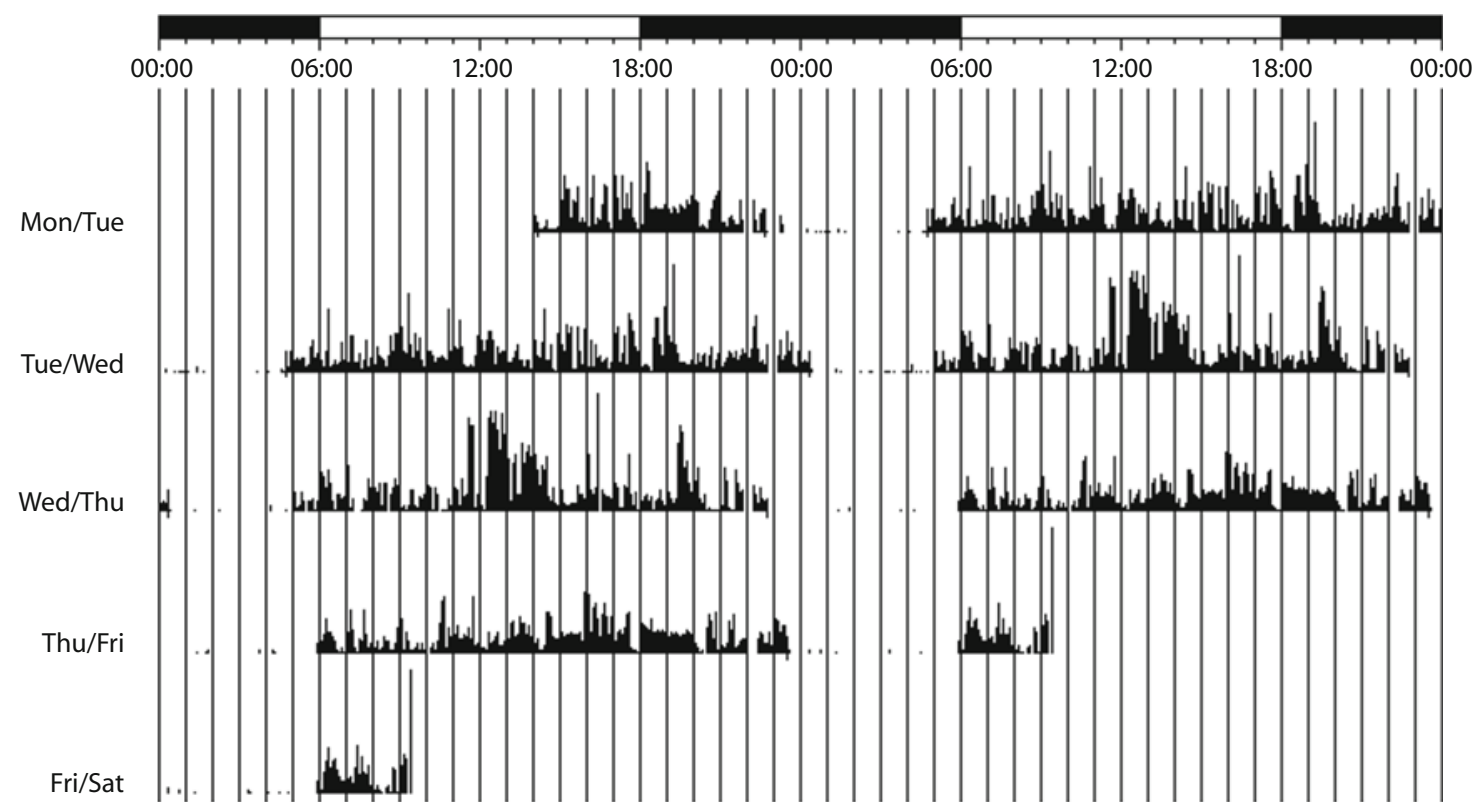

Figure 1. (A) Relationship between the agreement rate of wake and that of sleep. (B) Actogram of one case, showing high rates of agreement. 
Public Health, Nippon Medical School, for their technical support and advice. Correspondence concerning this article should be addressed to T. Kawada, Department of Hygiene and Public Health, Nippon Medical School, 1-1-5 Sendagi, Bunkyo-Ku, Tokyo 113-8602, Japan (e-mail: kawada@nms.ac.jp).

\section{REFERENCES}

Benson, K., Friedman, L., Noda, A., Wicks, D., Wakabayashi, E., \& YesaVAGE, J. (2004). The measurement of sleep by actigraphy: Direct comparison of 2 commercially available actigraphs in a nonclinical population. Sleep, 27, 986-989.

Carney, C. E., Lajos, L. E., \& Waters, W. F. (2004). Wrist actigraph versus self-report in normal sleepers: Sleep schedule adherence and self-report validity. Behavioral Sleep Medicine, 2, 134-143.

KAWADA, T. (2002). Effect of age on sleep onset time in rotating shift workers. Sleep Medicine, 3, 423-426.

Kawada, T., Kuroiwa, M., Sasazawa, Y., Suzuki, S., \& Tamura, Y. (2002). Sleep time monitoring by accelerometer in two subjects for 1 year. Journal of Sound \& Vibration, 250, 75-82.

Kawada, T., Shimizu, T., FujiI, A., Kuratomi, Y., Suto, S., Kanai, T., ET AL. (2008). Activity and sleeping time monitored by an accelerometer in rotating shift workers. Work, 30, 157-160.

KaWAdA, T., \& SUzUKI, S. (2002). Monitoring sleep hours using a sleep diary and errors in rotating shiftworkers. Psychiatry \& Clinical Neuroscience, 56, 213-214.
Kawada, T., Xin, P., Kuroiwa, M., Sasazawa, Y., SuZuki, S., \& TAMURA, Y. (2001). Habituation of sleep to road traffic noise as determined by polysomnography and accelerometer. Journal of Sound \& Vibration, 242, 169-178.

Kryger, M. H., Roth, T., \& Dement, W. C. (Eds.) (2005). Principles and practice of sleep medicine (4th ed.). Philadelphia: Saunders.

Lockley, S. W., Skene, D. J., \& Arendt, J. (1999). Comparison between subjective and actigraphic measurement of sleep and sleep rhythms. Journal of Sleep Research, 8, 175-183.

Paquet, J., KawinsKa, A., \& CARrier, J. (2007). Wake detection capacity of actigraphy during sleep. Sleep, 30, 1362-1369.

SADEH, A. (1995). Activity-based measures of sleep. In M. A. Carskadon (Ed.), Encyclopedia of sleep and dreaming (pp. 4-6). New York: Macmillan.

Sadeh, A., Sharkey, K. M., \& Carskadon, M. A. (1994). Activitybased sleep-wake identification: An empirical test of methodological issues. Sleep, 17, 201-207.

Werner, H., Molinari, L., Guyer, C., \& Jenni, O. G. (2008). Agreement rates between actigraphy, diary, and questionnaire for children's sleep patterns. Archives of Pediatrics \& Adolescent Medicine, 162, 350-358.

(Manuscript received June 10, 2008; revision accepted for publication June 19, 2008.) 